\title{
INTEGRAL upper limits on a bright X-ray flare from Sgr $A^{\star}$
}

\author{
Guillaume Trap ${ }^{* a, b}$, Andrea Goldwurm ${ }^{a, b}$, Régis $\operatorname{Terrier}^{b}$, Guillaume Bélanger ${ }^{c}$, \\ Farhad Yusef-Zadeh ${ }^{d}$ and Fulvio Melia ${ }^{e}$ \\ a Service d'Astrophysique (SAp) / IRFU / DSM / CEA Saclay, Bât. 709, 91191 Gif-sur-Yvette \\ Cedex, France \\ ${ }^{b}$ AstroParticule \& Cosmologie (APC) / Université Paris VII / CNRS / CEA / Observatoire de \\ Paris, Bât. Condorcet, 75205 Paris Cedex 13, France \\ ${ }^{c}$ European Space Agency (ESA) / ESAC, P.O. Box 78, Villanueva de la Canada, 28691 Madrid, \\ Spain \\ ${ }^{d}$ Department of Physics and Astronomy / Northwestern University, Evanston, Illinois 60208, USA \\ ${ }^{e}$ Department of Physics and Steward Observatory / The University of Arizona, Tucson, Arizona \\ 85721, USA
}

E-mail: trapeapc.univ-paris7.fr, andrea.goldwurmecea.fr, terriereapc.univ-paris7.fr, gbelangeresciops.esa.int, zadeh@northwestern.edu, melia@physics.arizona.edu

$\mathrm{Sgr} \mathrm{A}^{\star}$ is the supermassive black hole housed at the Galactic Center. Its non-thermal X-ray counterpart is variable and exhibits rapid flares about once per day. Besides, a soft $\gamma$-ray source discovered by INTEGRAL apparently coincident with Sgr A*, IGR J17456-2901, might be related to the X-ray emission. To gain more indsight on the possible links between X-rays and $\gamma$-rays, a new joint XMM-Newton/INTEGRAL campaign on the Galactic Center was performed in April 2007. Here, we report the INTEGRAL $\sim 100 \mathrm{ks}$ long observation of this campaign. For the first time, a bright X-ray flare from Sgr $A^{\star}$ detected by XMM-Newton $\left(\sim 16.1 \times 10^{-12} \mathrm{erg} \mathrm{s}^{-1} \mathrm{~cm}^{-2}\right.$, 2-10 keV) fell into INTEGRAL observing time. However, no evidence for variability was found by INTEGRAL/ISGRI, neither in the $20-40$ nor in the $40-100 \mathrm{keV}$ band, for which we place $3 \sigma$ upper limits on the flare's flux of 3.63 and $3.60 \times 10^{-11} \mathrm{erg} \mathrm{s}^{-1} \mathrm{~cm}^{-2}$, respectively.

7th INTEGRAL Workshop

September 8-11 2008

Copenhagen, Denmark

\footnotetext{
* Speaker.
} 


\section{Introduction}

From the discovery of a compact radio source, Sgr A*, at the Galactic Center (GC) in 1974 [1] to the near infrared (NIR) tracking of stars in Keplerian motion around Sgr $\mathrm{A}^{\star}$ three decades later $[2,3]$, the evidence for a $\sim 4 \times 10^{6} M_{\odot}$ black hole at the dynamical center of our galaxy gradually piled up (see [4] for a review and references therein).

Yet, the long quest for the high energy emission pertaining to the black hole has only been achieved recently, with the advent of high resolution and sensitivity in X-rays. Whereas galactic stellar black holes and active galactic nuclei are beacons in X-rays, Sgr A`s X-ray counterpart was resolved as a notably $\operatorname{dim}\left(2 \times 10^{33} \mathrm{erg} \mathrm{s}^{-1}\right)$ and slightly extended $\left(1^{\prime \prime}\right)$ point source with the Chandra satellite in 1999 [5]. One year later, the same instrument witnessed the source suddenly exhibiting a rapid X-ray flare, thereby temporarily increasing its X-ray luminosity by a factor of $\sim 50$ for $3 \mathrm{~h} \mathrm{[6]}$. A $10 \mathrm{~min}$ long substructure within the light curve of the eruption implies that this event took place close to the event horizon $\left(<15 R_{\mathrm{S}}\right.$, with $\left.R_{\mathrm{S}}=1.2 \times 10^{12} \mathrm{~cm}\right)$ with light travel arguments. Many other detections of X-ray flares with Chandra and XMM-Newton followed $[7,8,9,10]$, and established that the duty cycle of the black hole is nearly one X-ray flare per day. The origin of these events is still unclear, in spite of all the efforts aimed at the monitoring of flares in different energy ranges. In 2003, NIR flares from Sgr A ${ }^{\star}$ were indeed discovered with the VLT [11] and then confirmed with the Keck [12], but appear to occur more frequently than the $\mathrm{X}$-ray ones (several per day). Since then, numerous multiwavelength campaigns showed that an X-ray flare always comes along simultaneously with a NIR one $[13,14,15,16]$ and also possibly with a delayed submm one [17, 18, 19] caused by hot plasma expansion [20].

Above $20 \mathrm{keV}$, repeated surveys of the heart of the Milky Way in hard X-rays with the INTEGRAL satellite unveiled a persistent pointlike source compatible with $\mathrm{Sgr} \mathrm{A}^{\star}$ location (within the $1^{\prime}$ error radius), called IGR J17456-2901 [21, 22]. The nature of the source is still uncertain [44], and a possible association with the supermassive black hole remains conceivable. Given the limited angular resolution of the soft $\gamma$-ray telescope IBIS/ISGRI onboard INTEGRAL ( $\sim 13^{\prime}$ FWHM), the best way to unequivocally identify the mysterious source IGR J17456-2901 with Sgr A* would be the detection of correlated variability between X-rays and soft $\gamma$-rays. Therefore, we conducted an extensive and coordinated multiwavelength campaign in spring 2007, involving in particular the XMM-Newton and INTEGRAL satellites, for the high energies, as well as the VLT/NACO and $V L T /$ VISIR ground instruments, to cover the NIR and MIR part of the spectrum, respectively. The results of the infrared observations are beyond the scope of this article and will be reported in forthcoming papers [24, 25]. Hereafter, we concentrate solely on high energy results. We briefly repeat the published XMM-Newton's findings [26] in paragraph 2.1, use them to analyse INTEGRAL's observations in paragraph 2.2 and discuss them in paragraph 3.

Throughout this paper we adopt a GC distance of $8 \mathrm{kpc}$ [27] and a black hole mass of $4 \times$ $10^{6} M_{\odot}$ [28], for which the Schwarzschild radius is $R_{\mathrm{S}}=1.2 \times 10^{12} \mathrm{~cm}$.

\section{Observations and results}

\subsection{XMM-Newton}

The XMM-Newton satellite [29] was pointed towards the GC during $\sim 2.5$ consecutive revolu- 
tions, from March $30^{\text {th }}$ to April $4^{\text {th }} 2007$ (see fig.1, bottom image). The data of the EPIC cameras $[30,31]$ were processed and analyzed through the procedure described in [26]. On April $4^{\text {th }}$, a high level of flaring activity from Sgr $\mathrm{A}^{\star}$ was eventually caught. A bright flare-the second brightest ever recorded ( $\sim 100$ times the quiescent level) in the X-ray band $(2-10 \mathrm{keV})$ —was rapidly followed by three moderate ones. The bright event lasted $\sim 1 \mathrm{~h}$; its light curve has a rather symmetrical morphology and no apparent substructures (see fig.2). From a spectral point of view, this outburst was rather soft. Indeed, the best fit to the data with an absorbed power-law model, including dust scattering, yields the following parameters: a spectral photon index $\Gamma=2.3 \pm 0.3$ $\left(N(E) \propto E^{-\Gamma}\right)$ and a column density $N_{\mathrm{H}}=12 \pm 2 \times 10^{22} \mathrm{~cm}^{-2}$. The unabsorbed peak flux of the flare was $16 \pm 3 \times 10^{-12} \mathrm{erg} \mathrm{s}^{-1} \mathrm{~cm}^{-2}$, which translates to a luminosity of $2.4 \pm 4 \times 10^{35} \mathrm{erg} \mathrm{s}^{-1}$ at the GC distance. Note that this luminous eruption was still $\sim 9$ orders of magnitude below the Eddington luminosity for a supermassive black hole of this kind.

As pointed out by Porquet et al. [26], this rapid train of flares in just a few hours challenges disruption mechanisms as the origin of the outbursts, since they rely on temporary storage of mass/energy. This energy should indeed be released at once during the outburst, with a radiation efficiency of a few percent. But, the weak accretion rate of the black hole seems insufficient $\left(\sim 10^{16-17} \mathrm{~g} \mathrm{~s}^{-1}\right.$, [4]) to accumulate the required energy on such short timescales. In contrast, scenarios based on the stochastic infall and tidal disruption of gas clumps [32] or small bodies do not encounter this issue.

\subsection{INTEGRAL}

The INTEGRAL satellite [33] monitored the GC in parallel to XMM-Newton in April 2007, for a total effective exposure at $\mathrm{Sgr} \mathrm{A}^{\star}$ position of $\sim 212 \mathrm{ks}$ for IBIS/ISGRI $(20-100 \mathrm{keV})[34,35]$ and $\sim 46 \mathrm{ks}$ for JEM-X 1 (3-20 keV) [36] ${ }^{1}$. Measurements were spread over two consecutive revolutions, 545 and 546, from 2007-04-01 12:58:00 to 2007-04-02 21:32:34 and 2007-04-03 11:48:14 to 2007-04-04 20:26:59 (UT), respectively. In total, data of 74 individual pointings (science windows, ScW) were acquired, lasting $\sim 2930 \mathrm{~s}$ each. The whole dataset was reduced with OSA 7.0, the Offline Science Analysis Software, distributed by the INTEGRAL Science Data Center (ISDC) [37], with algorithms described in [38] for IBIS/ISGRI.

\section{Upper limits on the flare}

To search for a counterpart above $20 \mathrm{keV}$ of Sgr A April $^{\text {th }} \mathrm{X}$-ray flare, we selected the two consecutive ScW of ISGRI that covered the flare time interval, 054600220010 and 054600230010 , and produced a combined mosaic of the individual images, in two energy bands: 20-40 and 40-100 keV. None of these mosaics contained a distinctive source at the position of the black hole. Hence, no high energy counterpart of the flare was found. By considering the variance in $\mathrm{Sgr} \mathrm{A}^{\star}$ pixel, we derive $3 \sigma$ upper limits on the flare of 1.17 and $1.11 \mathrm{cts} \mathrm{s}^{-1}$ in the $20-40$ and 40 $100 \mathrm{keV}$ bands, respectively. Assuming a power-law spectral shape of index $\Gamma=2.3$ (paragraph 2.1 ), these rates convert to fluxes of 3.63 and $3.60 \times 10^{-11} \mathrm{erg} \mathrm{s}^{-1} \mathrm{~cm}^{-2}$, respectively.

\footnotetext{
${ }^{1}$ Compared to IBIS, JEM-X field of view is narrower, so given the rectangular dithering pattern used $(5 \times 5$ pointings: 24 off-source +1 on-source), the GC was invisible to JEM-X most of the time, which explains the discrepancy in the exposures.
} 


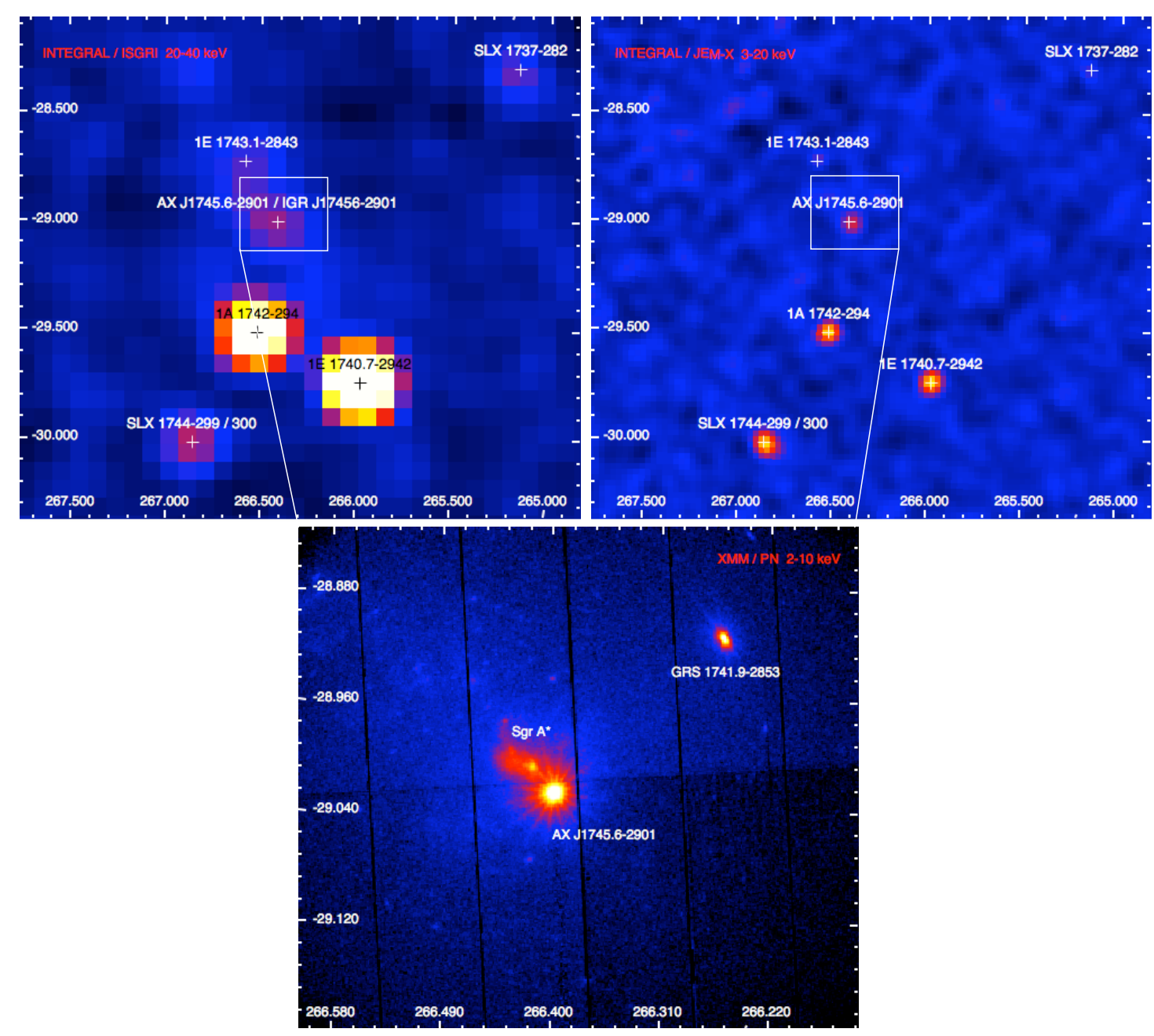

Figure 1: Multiwavelength views of the inner region of the galaxy in April 2007, in R.A. $\left(^{\circ}\right)$ horizontally and Dec. $\left({ }^{\circ}\right)$ vertically. Top, left: INTEGRAL/ISGRI significance mosaic of the whole campaign in the $20-40 \mathrm{keV}$ band ( $212 \mathrm{ks}$ exposure). The galactic plane runs from upper left to bottom right and each pixel size is equivalent to $\sim 5^{\prime}$. Top, right: INTEGRAL/JEM-X 1 significance mosaic of the whole campaign in the 3-20 keV band with the same angular scale ( $\sim 46 \mathrm{ks}$ exposure). Bottom: Magnified view of the GC in the $0.1-10 \mathrm{keV}$ range with XMM-Newton/PN (revolution $\mathrm{n}^{\circ} 1340,97.6 \mathrm{ks}$ exposure). Sgr $\mathrm{A}^{\star}$ stands out in the middle of the Sgr A complex, because this observation contains several flares from the vicinity of the black hole, enhancing its average luminosity (see text).

Regarding JEM-X 1 data in the $3-20 \mathrm{keV}$ band, we report similar results. There was no detection in the combined mosaic, and given a flare duration of $\sim 3000 \mathrm{~s}$, the sensitivity curves of JEM-X [39] provide $5 \sigma$ upperlimits of 10 and $7 \times 10^{-11} \mathrm{erg} \mathrm{s}^{-1} \mathrm{~cm}^{-2}$ in the 3-10 and 10-25 keV energy ranges, respectively.

\section{Light curves and mosaics}

On fig.2 (top panel), we plotted the ISGRI light curves of the pixel at the position of Sgr A*, built with individual ScW. It is noteworthy that no source was significantly detected in any individual exposure. 


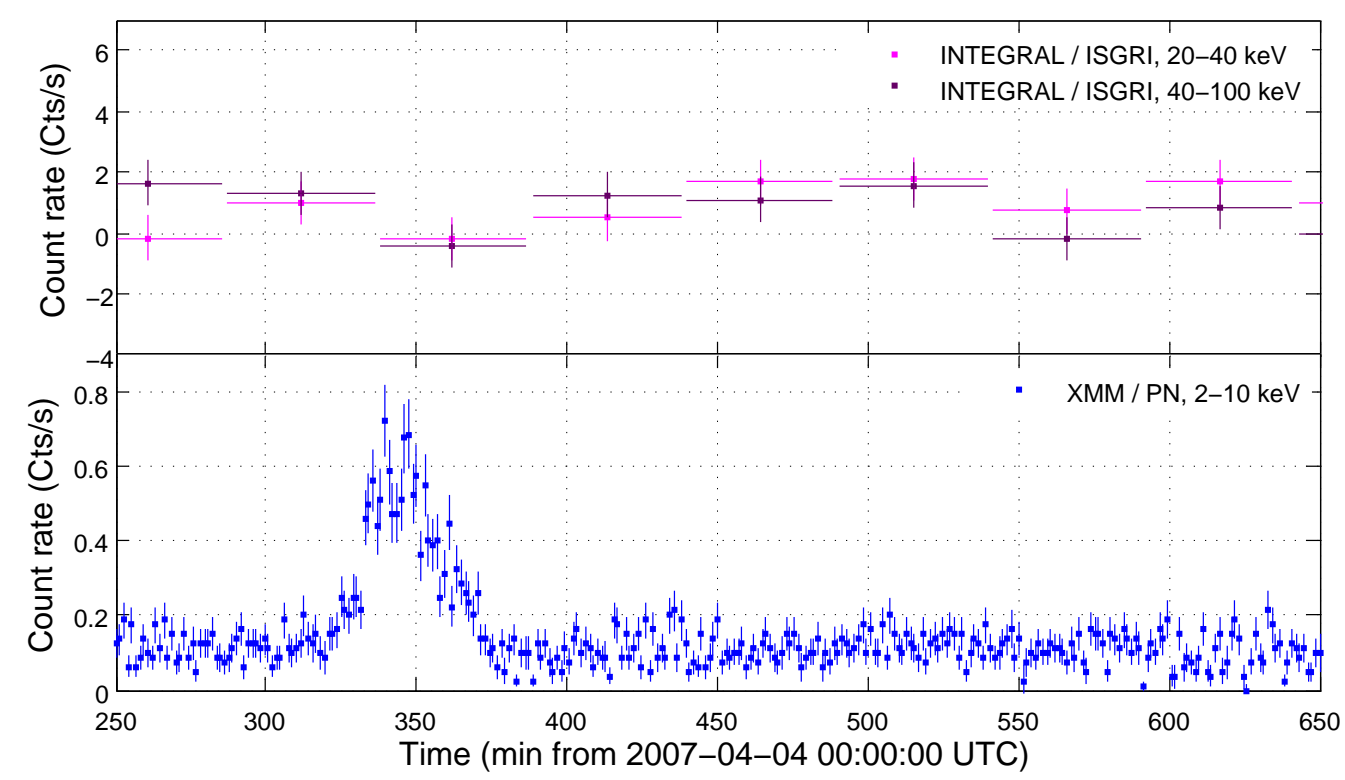

Figure 2: Top: Light curves of the pixel at the position of Sgr $\mathrm{A}^{\star}$ with a time bin of $\sim 2900 \mathrm{~s}$. The signal to noise ratio within each bin is weak and is primarily due to IGR J17456-2901 and incidentally to AX J1745.62901 (see text). Bottom: Background substracted light curve of a 10" radius region centered on Sgr A ${ }^{\star}$ with a time bin of $80 \mathrm{~s}$. The non flaring parts do not correspond uniquely to $\mathrm{Sgr} \mathrm{A}^{\star}$ quiescent emission, but include diffuse emission and point sources less than $10^{\prime \prime}$ from the black hole that cannot be resolved by $X M M-N e w t o n$ as well.

In contrast to individual ScW, the total ISGRI and JEM-X 1 mosaics of the observation dataset both reveal a significant excess at the position of $\mathrm{Sgr} \mathrm{A}^{\star}$ (see fig.1, top panels). The significances of these signals are both $13.7 \sigma$. Considering the XMM-Newton $2-10 \mathrm{keV}$ image (fig.1, bottom panel), we see that the transient neutron star low-mass X-ray binary AX J1745.6-2901, located just $1.5^{\prime}$ from $\mathrm{Sgr}^{\star}{ }^{\star}$ in projection, was markedly the dominant source of the region. Given JEM-X angular resolution of $3^{\prime}$ (FWHM), we can safely associate its 3-20 keV excess with the binary. In the ISGRI mosaic (fig.1, top, left), the point spread function (PSF) is 13' (FWHM), and so does not allow us to disentangle AX J1745.6-2901 from IGR J17456-2901, the persistent hard X-ray source discovered by INTEGRAL/ISGRI ${ }^{2}$ [21, 22]. To assess the contribution of the transient binary to the ISGRI signal though, we compared the April 2007 20-40 keV mosaic with another equivalent GC map, constructed with data spanning over 4 months, from August to November 2006. During this latter period, we know for sure that the transient binary was in quiescence and undetected at high energies, thanks to a regular Swift/XRT monitoring of the GC [40]. So, the total count rate of $0.86 \pm 0.03 \mathrm{cts} \mathrm{s}^{-1}$ we measured in the central pixel of the excess in the 2006 mosaic, can be entirely attributed to IGR J17456-2901. In April 2007, we found that the total count rate increased to $0.97 \pm 0.07 \mathrm{cts} \mathrm{s}^{-1}$, so that, assuming IGR J17456-2901 remained constant, the photons from the 20-40 keV excess visible in fig. 1 (top, left) came at $~ 90 \%$ from IGR J17456-2901 and 10\% from AX J1745.6-2901.

\footnotetext{
${ }^{2}$ Notice that IGR J17456-2901 has not been significantly detected with JEM-X yet.
} 


\section{Discussion}

This is the first time INTEGRAL was gazing the GC during a period of known flaring activity from Sgr A ${ }^{\star}$. Last X-ray $/ \gamma$-ray coordinated campaigns in 2004 were, indeed, inconclusive, since the X-ray flares detected then by XMM-Newton occured at times when INTEGRAL was crossing the radiation belts with all its intruments in standby mode [22].

As indicated above, we did not identify any $\gamma$-ray counterpart of the intense $\mathrm{X}$-ray flare from April $4^{\text {th }}$. This proves once again that $\operatorname{Sgr}^{\star}$ does not release the bulk of its emission in soft $\gamma$ rays [41]. This result is also somewhat reminescent of the 2005 Chandra/HESS joint campaign, which demonstrated that the TeV source of the GC, HESS J1745-290, stayed still during an X-ray outburst seen by Chandra [42].

On fig.3, we display the broad band quiescent spectral energy distribution (SED) of Sgr A* in dark gray. We also overplot in colors (blue, magenta and purple) the spectral information on the 2007 April $^{\text {th }}$ flare we gathered at high energies. Clearly, the INTEGRAL/ISGRI upper limits are not extremely contraining for the emission models invoked to interpret this event. As will be shown in [24, 25], when the NIR and MIR measurements of this flare are taken into account, simple synchrotron or synchrotron self-Compton emission models that fit the X-ray data, do not violate the $\gamma$-ray constraints. The reason for this is that the X-ray spectral shape of the flare is soft (with a negative slope of -0.3 in $v F_{V}$ ). By simply extrapolating the $\mathrm{X}$-ray power-law, one expects fluxes of 3.9 and $4.1 \times 10^{-12} \mathrm{erg} \mathrm{s}^{-1} \mathrm{~cm}^{-2}$ in the $20-40$ and $40-100 \mathrm{keV}$, respectively. These expected values are roughly one order of magnitude below the $3 \sigma$ constraints worked out above, which suggest that the next generation of hard X-ray focusing instruments, like Simbol-X [43], will be able to extend spectral measurements on a flare of the GC supermassive black hole above $20 \mathrm{keV}$ [44].

Concerning IGR J17456-2901, it was relatively improbable to find it flare up in April 2007, based on the long ISGRI exposures targeted at the source in 2003 and 2004. These did not reveal any sign of variability on any timescale [22], even though a poor correction of the background induced artificial temporal artefacts in the early light curves of IGR J17456-2901 [21]. Note, however, that variability on a single $\mathrm{ScW}$ duration basis cannot really be excluded, since this time interval is too short to convincingly detect the source IGR J17456-2901.

The provenance of IGR J17456-2901 thus remains enigmatic. We showed that the activity of the luminous transient binary AXJ1745.6-2901 did not amount to more than $\sim 10 \%$ of the total 20-40 keV flux of IGR J17456-2901, contrary to what was alluded to in [45]. The absence of variability and the fact that the flux of IGR J17456-2901 is two orders of magnitude above the quiescent emission of $\mathrm{Sgr} \mathrm{A}^{\star}$ as measured by Chandra, supports the idea that the hard X-ray photons visible in INTEGRAL's mosaics are unlikely to be produced in the inner region of the accretion/ejection flow around the black hole. Instead, these photons should arise from a diffuse, and yet compact (a few arcminutes), zone, or maybe result from the sum of unresolved hard Xray point sources [46]. A possible connection between IGR J17456-2901 and HESS J1745-290 is another option. Hinton and Aharonian [47] proposed that $\sim 10-100 \mathrm{TeV}$ electrons permeating the inner 20 pc may be responsible for the combined XMM-Newton/INTEGRAL spectrum of the central $8^{\prime}$ radius region [22] via synchrotron emission, as well as HESS J1745-290 through inverse Compton (IC) processes. These authors favor the pulsar wind nebula candidate G 359.95-0.04 [48] 


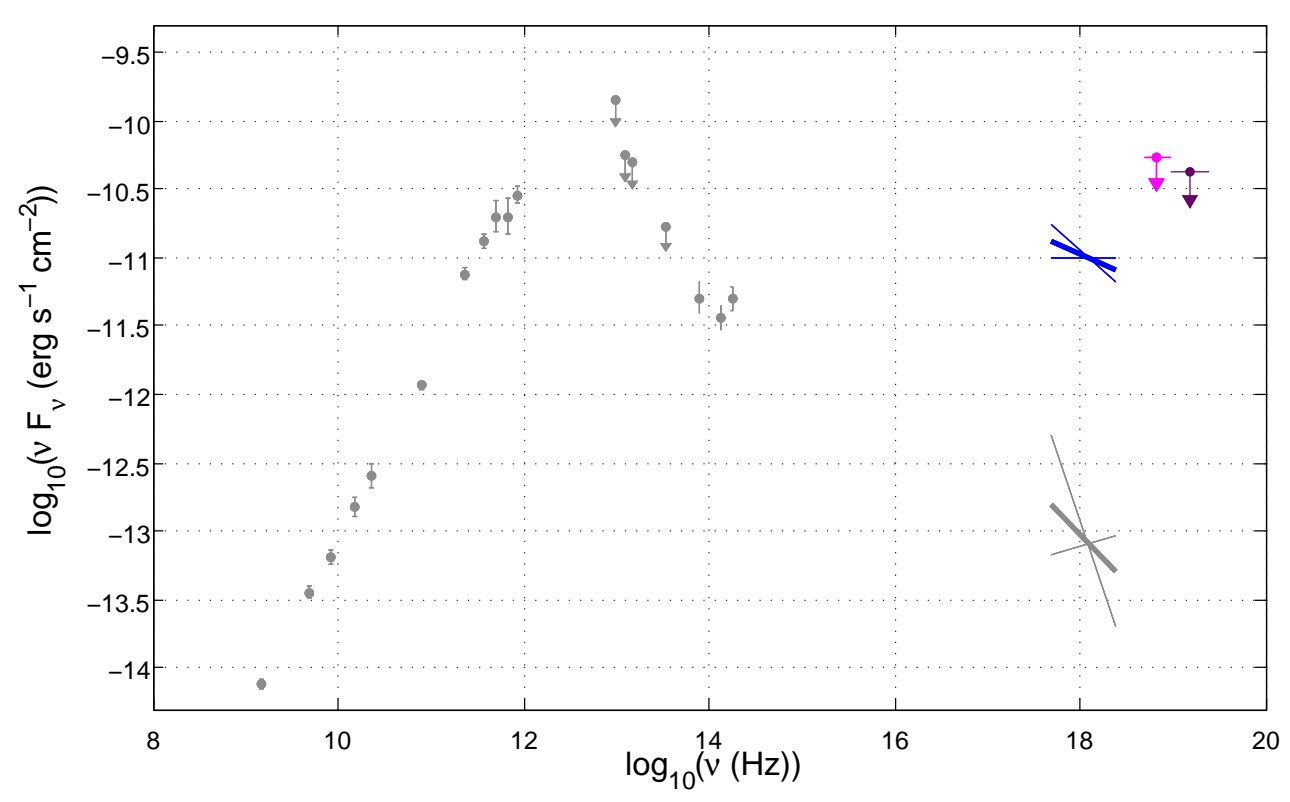

Figure 3: Spectral energy distribution of Sgr A ${ }^{\star}$. Dark gray measurements correspond to the quiescent state. Radio and submm points are extracted from [49, 50, 17], FIR and MIR upperlimits from [51, 52, 14, 53], NIR points from [11] and the X-ray bow tie from [5]. The XMM-Newton spectrum of the April $4^{\text {th }}$ flare and the INTEGRAL/ISGRI upper limits are overplotted with the same color coding as in figure 2.

as the X-ray counterpart of HESS J1745-290, though. In their scenario, the TeV photons come about in the compact nebula, just 0.3 pc from Sgr $\mathrm{A}^{\star}$, by the IC boosting of ambient photons by relativistic electrons originating from the pulsar. Nevertheless, IGR J17456-2901 does not fit within this frame, as its flux is too high to be the simple hard X-ray extension of G 359.95-0.04 soft $\mathrm{X}$-ray flux as determined by Chandra [48]. Again, the increased angular resolution and sensitivity in the hard X-ray range of the next generation of instruments will also help address the question of IGR J17456-2901 true nature.

Acknowledgments GT acknowledges M. Falanga, D. Götz and J. Chenevez for useful discussions about the INTEGRAL data analysis. Part of this work was supported by the french Agence Nationale pour la Recherche through grant ANR-06-JCJC-0047. INTEGRAL is an ESA project with instruments and science data center funded by ESA member states (especially the PI countries: Denmark, France, Germany, Italy, Switzerland, and Spain), the Czech Republic, and Poland, and with the participation of Russia and the US. The XMM-Newton project is an ESA Science Mission with instruments and contributions directly funded by ESA Member States and the USA (NASA).

\section{References}

[1] B. Balick \& R.L. Brown, Intense sub-arcsecond structure in the galactic center, ApJ 194 (265) 1974

[2] R. Schödel et al., A star in a 15.2-year orbit around the supermassive black hole at the centre of the Milky Way, Nat 419 (694) 2002

[3] A. Ghez et al., The First Measurement of Spectral Lines in a Short-Period Star Bound to the Galaxy's Central Black Hole: A Paradox of Youth, ApJ 586 (L127) 2003 
[4] F. Melia, The Galactic Supermassive Black Hole, Princeton University Press 2007 [ISBN-13: 978-0691131290]

[5] F.K. Baganoff et al., Chandra X-Ray Spectroscopic Imaging of Sagittarius $A^{*}$ and the Central Parsec of the Galaxy, ApJ 591 (891) 2003

[6] F.K. Baganoff et al., Rapid X-ray flaring from the direction of the supermassive black hole at the Galactic Centre, Nat 413 (45) 2001

[7] F.K. Baganoff et al., Multiwavelength Monitoring of Sgr $A^{*}$ During Chandra Observations of Multiple X-ray Flares, BAAS 34 (1153) 2003

[8] A. Goldwurm et al., A New X-Ray Flare from the Galactic Nucleus Detected with the XMM-Newton Photon Imaging Cameras, ApJ 584 (751) 2003

[9] D. Porquet et al., XMM-Newton observation of the brightest X-ray flare detected so far from Sgr $A^{*}$, $A \& A 407$ (L17) 2003

[10] G. Bélanger et al., Repeated X-ray Flaring Activity in Sagittarius A*, ApJ 635 (1095) 2005

[11] R. Genzel et al., Near-infrared flares from accreting gas around the supermassive black hole at the Galactic Centre, Nat 425 (934) 2003

[12] A. Ghez et al., Variable Infrared Emission from the Supermassive Black Hole at the Center of the Milky Way, ApJ 601 (L159) 2004

[13] A. Eckart et al., First simultaneous NIR/X-ray detection of a flare from Sgr A*, A\&A 427 (1) 2004

[14] A. Eckart et al., The flare activity of Sagittarius $A^{*}$. New coordinated mm to X-ray observations, A\&A 450 (535) 2006

[15] A. Eckart et al., Polarized NIR and X-ray flares from Sagittarius $A^{*}, A \& A 479$ (625) 2008

[16] F. Yusef-Zadeh et al., A Multiwavelength Study of Sgr A*: The Role of Near-IR Flares in Production of X-Ray, Soft $\gamma$-Ray, and Submillimeter Emission, ApJ 644 (198) 2006

[17] D. Marrone et al., An X-Ray, Infrared, and Submillimeter Flare of Sagittarius A*, ApJ 682 (373) 2008

[18] F. Yusef-Zadeh et al., Simultaneous Chandra, CSO and VLA Observations of Sgr A*: The Nature of Flaring Activity, ApJ 682 (361) 2008

[19] A. Eckart et al., Simultaneous NIR/sub-mm observation of flare emission from SgrA*, subm. to A\&A 2008 [A

[20] F. Yusef-Zadeh et al., Flaring Activity of Sagittarius $A^{*}$ at 43 and 22 GHz: Evidence for Expanding Hot Plasma, ApJ $6 \mathbf{6 0}$ (189) 2006

[21] G. Bélanger et al., Detection of Hard X-Ray Emission from the Galactic Nuclear Region with INTEGRAL, ApJ 601 (L163) 2004

[22] G. Bélanger et al., A Persistent High-energy Flux from the Heart of the Milky Way: INTEGRAL's View of the Galactic Center, ApJ 636 (275) 2006

[23] A. Goldwurm, A multiwavelength view of high-energy processes in the Galactic Center region, Gamma 2008 Heidelberg Proceedings 2008

[24] K. Dodds-Eden, Evidence for X-ray synchrotron emission from simultaneous mid-IR to X-ray observations of a strong Sgr A* flare, subm. to ApJ 2008

[25] G. Trap, in prep., to be subm. to ESASP 2008 
[26] D. Porquet et al., X-ray hiccups from Sagittarius $A^{*}$ observed by XMM-Newton. The second brightest flare and three moderate flares caught in half a day, A\&A 488 (549) 2008

[27] M.J. Reid, The distance to the center of the Galaxy, ARA\&A 31 (345) 1993

[28] A. Ghez et al., Measuring Distance and Properties of the Milky Way's Central Supermassive Black Hole with Stellar Orbits, Subm. to ApJ 2008,[ArXiv: 0808 -2870]',

[29] F. Jansen et al., XMM-Newton observatory. I. The spacecraft and operations, A\&A 365 (L1) 2001

[30] L. Strüder et al., The European Photon Imaging Camera on XMM-Newton: The pn-CCD camera, $A \& A 365$ (L18) 2001

[31] M.J.L. Turner et al., The European Photon Imaging Camera on XMM-Newton: The MOS cameras : The MOS cameras, A\&A 365 (L27) 2001

[32] M. Tagger \& F. Melia, A Possible Rossby Wave Instability Origin for the Flares in Sagittarius $A^{*}$, ApJ 636 (L33) 2006

[33] C. Winkler et al., The INTEGRAL mission, A\&A 411 (L1) 2003

[34] P. Ubertini et al., IBIS: The Imager on-board INTEGRAL, A\&A 411 (L131) 2003

[35] F. Lebrun et al., ISGRI: The INTEGRAL Soft Gamma-Ray Imager, A\&A 411 (L141) 2003

[36] N. Lund et al., JEM-X: The X-ray monitor aboard INTEGRAL, A\&A 411 (L231) 2003

[37] T.J.-L. Courvoisier et al., The INTEGRAL Science Data Centre (ISDC), A\&A 411 (L53) 2003

[38] A. Goldwurm et al., The INTEGRAL/IBIS scientific data analysis, A\&A 411 (L223) 2003

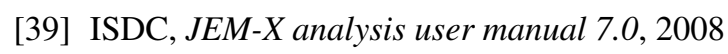

[40] N. Degenaar and R. Wijnands, The behavior of sub-luminous X-ray transients near the Galactic Center as observed using the X-ray telescope aboard Swift, Subm. to A\&A 2008 [ArXiv:0807.3458],

[41] A. Goldwurm, Evidence against a Massive Black-Hole at the Galactic Center, Nat. 371 (589) 1994

[42] J. Hinton et al., Simultaneous H.E.S.S. and Chandra observations of Sgr $A^{*}$ during an X-ray flare, ICRC 2007![ArXiv:0710.1537]!

[43] P. Ferrando et al., The Simbol-X mission, MSAI 79 (19) 2008

[44] A. Goldwurm, Observing the Super-Massive Black Hole of the Galactic center with Simbol-X, MSAI 79 (81) 2008

[45] M. Revnivstev et al., Hard X-ray view of the past activity of Sgr $A^{*}$ in a natural Compton mirror, A\&A 425 (L49) 2004

[46] M. Revnivstev et al., Origin of the Galactic ridge X-ray emission, A\&A 452 (L169) 2006

[47] J. Hinton \& F. Aharonian, Inverse Compton Scenarios for the TeV Gamma-Ray Emission of the Galactic Center, ApJ 657 (302) 2007

[48] D.Q. Wang at al., G359.95-0.04: an energetic pulsar candidate near Sgr A*, MNRAS 367 (937) 2006

[49] J.-H. Zhao et al., Radio Variability of Sagittarius A*-a 106 Day Cycle, ApJ 547 (L29) 2001

[50] R. Zylka et al., Anatomy of the Sagittarius A complex. 4: Sgr A* and the Central Cavity revisited, A\&A 297 (83) 1995 
[51] C.M. Telesco et al., 10-30 Micron Maps of the Central 5 Parsecs of the Galaxy: Heating of the Cavity and Neutral Gas Disk, ApJ 456 (541) 1996

[52] A. Cotera et al., Mid-Infrared Imaging of the Central Parsec with Keck, ASPC 186 (240) 1999

[53] R. Schödel et al., The possibility of detecting Sagittarius $A^{*}$ at $8.6 \mu \mathrm{m}$ from sensitive imaging of the Galactic center, A\&A 462 (L1) 2007 